\title{
Antineoplastic Alkylating Agent
}

National Cancer Institute

\section{Source}

National Cancer Institute. Antineoplastic Alkylating Agent. NCI Thesaurus. Code C1590.

An antineoplastic agent that replaces hydrogen atom(s) in nucleophilic moieties with alky radical(s), hindering proper function. Alkylating agents exhibit cytotoxic effects through the alkylation of DNA, resulting in strand cross-linking, ultimately inhibiting DNA replication and cancer cell growth. 